\title{
ESTATÍSTICA MULTIVARIADA NA DISCRIMINAÇÃO DE RAÇAS AMAZÔNICAS DE PUPUNHEIRAS (Bactris gasipaes Kunth) EM MANAUS (BRASIL) ${ }^{1}$
}

\author{
JORGE HUGO IRIARTE MARTEL ${ }^{2}$, ANTONIO SERGIO FERRAUDO ${ }^{3}$, JOSÉ ROBERTO MÔRO ${ }^{3}$, DILERMANDO \\ PERECIN $^{3}$
}

\begin{abstract}
RESUMO - As raças e populações de pupunheiras ao longo dos rios Amazonas e Solimões apresentam grande variabilidade genética ainda não totalmente caracterizada. Neste estudo, foram aplicadas técnicas estatísticas multivariadas a 15 descritores morfológicos numa tentativa de caracterizar, morfometricamente, três raças existentes ao longo da Bacia desses rios. As três análises em conjunto permitiram uma discriminação das raças, mostrando também que os descritores mais importantes nessa seleção foram: número de espigas, comprimento da ráquis, peso do fruto, espessura das cascas, facilidade para descascar os frutos, peso das cascas, sabor dos frutos, espessura da polpa, distância morfológica dos frutos e peso da semente.
\end{abstract}

Termos para indexação: descritores, análise multivariada, análise de componentes principais, análise discriminante, análise de agrupamento.

\section{MULTIVARIATE ANALYSIS ON DISCRIMINATION OF AMAZONIC LANDRACES OF PEACH PALM (Bactris gasipaes Kunth) IN MANAUS, BRAZIL}

\begin{abstract}
Landraces and populations of peach palm growing along the Amazon and Solimões rivers present great genetic variability, that it was not totally characterized yet. In the present study, multivariate techniques of cluster analysis, principal components analysis and discriminant analysis, were applied to fifteen morphological descriptors with the objective of morphometric characterizing three existing landraces of this plant along these rivers basins. The three analysis together allowed to discriminate the studied landraces, showing that the most important descriptors were: numbers of ears per raceme, rachis length, fruit weight, thickness of fruit bark, facility to peel fruits, weight of fruit bark, fruit flavor, pulp thickness, morphological distance of fruit and seed weight.
\end{abstract}

Index terms: Descriptors, multivariate analysis, principal component analysis, discriminant analysis and cluster analysis.

\section{INTRODUÇÃO}

A pupunha tem um potencial econômico e social muito grande como fonte de alimento para o homem e animais, sendo sem dúvida a palmeira mais importante na América pré-colombiana. É tão importante quanto o milho e a mandioca, sendo o principal cultivo dos ameríndios de um extenso território do trópico úmido e de algumas regiões do trópico seco (Mora Urpi, 1982; 1984). Mora Urpi et al. (1997) relataram que, na Amazônia, existem pelo menos oito raças primitivas de pupunheiras, e ao noroeste dos Andes, pelo menos mais cinco raças. Apresentaram a distribuição geográfica de mais 15 raças de pupunha, mostrando a existência das raças Pará, Solimões e Putumayo, embora os limites exatos entre essas raças não sejam claros, já que existe muita interseção de acessos entre as raças.

Dados de Mora Urpi \& Clement (1988) e Clement (2000), baseados em análise morfométrica, afirmam a existência de três raças ao longo da bacia amazônica. Trabalhos realizados com biologia molecular, para verificar a existência das três raças (Rodrigues, 2001; Clement et al., 2002; Souza et al., 2002), negam a existência da raça Solimões. Quando se deseja discriminar grupos ou populações, Sokal (1965) e Sneath \& Sokal (1973) afirmam que a seleção das características é fundamental. As técnicas de estatística multivariada têm a capacidade de estudar características como um conjunto sinergístico, oferecendo um método adequado a ser aplicado em estudos de raças e populações de pupunheiras.

As técnicas de análise de agrupamento têm como objetivo dividir um grupo original de observações em vários grupos, seguindo algum critério de similaridade ou dissimilaridade (Cruz \& Regazzi, 1994). Sneath \& Sokal (1973) relatam que os métodos de agrupamento mais utilizados são os seqüenciais, aglomerativos, hierárquicos e não superpostos. O dendrograma é um diagrama em forma de árvore que mostra a subdivisão dos grupos formados, buscando máxima homogeneidade entre os indivíduos no grupo e máxima heterogeneidade entre os grupos.

A caracterização morfológica de frutos e sementes tem sido utilizada para a delimitação de subfamílias e tribos de Crusiaceae (Mourão \& Beltratti, 2000); sendo assim, é importante a aplicação das técnicas estatísticas multivariadas, na tentativa de discriminar raças e populações de pupunha.

A descrição sistemática de uma espécie facilita ou possibilita o uso potencial do material genético, que é a meta principal dos Bancos de Germoplasma. Assim, neste estudo, foi utilizada a coleção de germoplasma de pupunha do Instituto Nacional de Pesquisas da Amazônia-INPA, visando a examinar a existência de três raças de pupunheiras ao longo dos rios Amazonas e Solimões.

A análise de agrupamento pode ser complementada com a análise de componentes principais, cujo objetivo é tentar explicar a estrutura de variância e co-variância das variáveis originais, construindo, mediante processo matemático, um conjunto menor de combinações lineares das variáveis originais que preserve a maior parte da informação fornecida por essas variáveis.

A análise discriminante multivariada também foi utilizada numa tentativa de classificar os locais que contêm as raças. O propósito deste trabalho é aplicar as três técnicas estatísticas multivariadas utilizando 15 descritores morfológicos em três raças de pupunha (Bactris gasipaes Kunth), em Manaus (Brasil), com o objetivo de caracterizá-las morfometricamente.

\section{MATERIAL E MÉTODOS}

O banco de germoplasma de pupunha do INPA está localizado no km 38 da Rodovia BR-174 (Manaus-Boa Vista-RR), no município de Manaus. Cada acesso de pupunha está representado por nove plantas de uma única matriz (progênie de meios-irmãos), em espaçamento de $5 \mathrm{x}$ $5 \mathrm{~m}$. O solo é um Latossolo Amarelo, textura pesada, localizado em um platô suave-ondulado, cuja vegetação original foi floresta alta tropical (Ranzani, 1980). O clima caracteriza-se como Afi, no esquema Köeppen (Ribeiro, 1976). A caracterização morfológica de pupunha foi realizada a partir de plantas com oito anos, com um cacho por planta e 10 frutos por cacho. Foi utilizada uma lista de descritores morfológicos, recomendada para pupunha por Clement (1986) e testada por Iriarte Martel (2002), sendo caracterizadas morfologicamente duzentas e sessenta plantas das raças 'Pará', 'Putumayo' e 'Solimões' (Tabela 1)

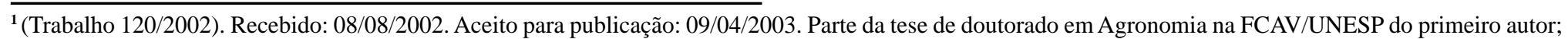
${ }^{2} \mathrm{Eng}^{\circ} \mathrm{Agr}^{\circ}$, Dr., Pesquisador do INPA, caixa postal 478, 69011-970, Manaus, AM <jhim@inpa.gov.br> .

${ }^{3}$ Prof. Dr. da FCAV/UNESP, Campus de Jaboticabal.Via de acesso Prof. Paulo Donato Castellane, km 5, 14870-000.<jrmoro@fcav.unesp.br>.
} 
TABELA 1- Número de acessos e plantas de 16 populações de pupunheiras das raças Pará, Putumayo e Solimões, existentes no INPA.

\begin{tabular}{lcc}
\hline Locais & Número de acessos & Número de plantas \\
\hline Totais Raça Pará & 22 & 67 \\
\hline Belém(PA) & 07 & 16 \\
\hline Santarém (PA) & 01 & 03 \\
\hline Rio Preto da Eva (AM) & 06 & 26 \\
\hline Manaus (AM) & 08 & 22 \\
\hline Totais Raça Putumayo & 45 & 149 \\
\hline Manacapuru (AM) & 01 & 04 \\
\hline Alto Solimões (AM) & 11 & 37 \\
\hline Santo A. do Içá (AM) & 03 & 07 \\
\hline Aldeia Vendaval (AM) & 05 & 32 \\
\hline São P. de Olivença (AM) & 06 & 28 \\
\hline Aldeia Umari-açu (AM) & 05 & 05 \\
\hline Benjamim Constant (AM) & 08 & 21 \\
\hline Tabatinga (AM) & 06 & 15 \\
\hline Totais Raça Solimões & 13 & 44 \\
\hline Coari (AM) & 01 & 12 \\
\hline Tefé (AM) & 04 & 07 \\
\hline Fonte Boa (AM) & 06 & 16 \\
\hline Jutaí (AM) & 02 & 09 \\
\hline
\end{tabular}

Os 15 descritores morfológicos foram: número de espigas por cacho (Nuesca), comprimento da ráquis (Comraq), distância morfológica dos frutos (Distan), peso dos frutos (Pesfru), adensamento dos frutos no cacho (Compca), espessura das cascas (Espcas), facilidade para descascar os frutos (Facilde), peso das cascas (Pescas), textura da polpa (Texpol), sabor dos frutos (Sabfru), espessura da polpa (Espend), peso das sementes (Peseme) e teores de Água, Óleo e Fibras. Foi verificada a consistência dos descritores entre as raças, através do teste Wilk'slambda. Para a aplicação das análises multivariadas, o conjunto de dados foi padronizado, ficando cada descritor com média nula e variância unitária. A análise de agrupamento foi processada segundo a metodologia proposta por Sneath \& Sokal (1973), e foi aplicada aos dados, utilizandose como coeficiente de semelhança entre pares de locais a distância euclidiana, que é um coeficiente de dissimilaridade, pois, quanto menor a distância entre dois locais, mais similares eles são, segundo as características consideradas. A estratégia de agrupamento adotada foi a Average Linkage (UPGMA - Unweighted Pair Group Method with Arithmetic Averages).

$\mathrm{Na}$ análise de componentes principais, a variância contida em cada componente principal é expressa pelos autovalores da matriz padronizada. O maior autovalor é associado ao primeiro componente principal, o segundo maior autovalor ao segundo componente principal, até que o menor autovalor esteja associado ao último componente principal, o que coloca os primeiros como os mais importantes. Sendo assim, os primeiros componentes principais explicam, geralmente, grande parte da variância das variáveis originais. Esta análise permitiu reduzir o espaço de variáveis originais ( 15 descritores) num conjunto menor (oito descritores), preservando o máximo da variabilidade original dos dados. A esse conjunto, aplicou-se a análise discriminante segundo a metodologia recomendada por Kleka (1975), Tabachnick \& Fidell (1983) e Engelman (1997). Como resultado desta análise, foram obtidas duas funções discriminantes que conseguiram reter $100 \%$ da variância inicial, permitindo uma reclassificação dos acessos. As análises foram processadas no software STATISTICA, versão 6,0.

\section{RESULTADOS E DISCUSSÃO}

A Figura 1 apresenta o resultado obtido na análise de agrupamento, mostrando a formação de três grupos. O grupo 1 é formado pelas populações de Manaus, Rio Preto da Eva, Belém, Santarém e Coari. Esse grupo é formado por populações da raça Pará com exceção à população
Coari, que é citada na literatura como pertencente à raça Solimões (MoraUrpi \& Clement, 1988). O grupo 2 é formado pelas populações de Vendaval, Alto Solimões, Tabatinga, São Paulo de Olivença, Benjamim Constant e Umari-açu, todas da raça Putumayo. O grupo 3 é formado pelas populações de Tefé, Jutaí e Fonte Boa, todas da raça Solimões. As populações de Manacapuru e de Santo Antonio do Içá não pertenceram a nenhum grupo. A população de Manacapuru é citada na literatura como pertencente à raça Pará, e Santo Antônio do Içá pertencente à raça Putumayo (Mora Urpi \& Clement, 1988).

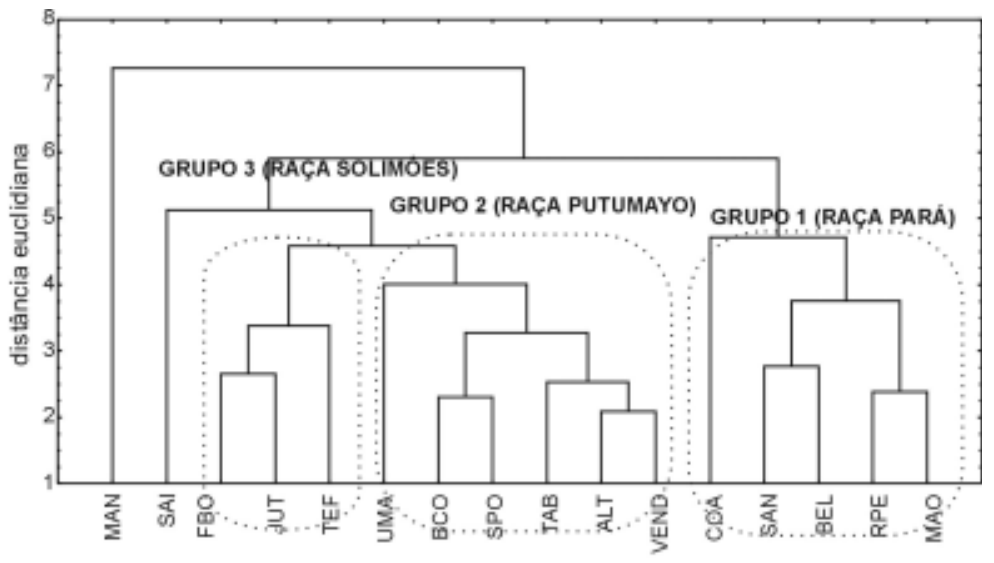

FIGURA 1- Classificação das populações das três raças de pupunha segundo a análise de agrupamento.

$\mathrm{Na}$ análise de componentes principais, foram considerados os dois primeiros componentes CP1 e CP2, que armazenaram 59,2\% da variância original dos descritores (Tabela 2).

TABELA 2- Correlação entre cada descritor e os respectivos componentes principais, para as raças Pará, Putumayo e Solimões, dos 15 descritores morfológicos.

\begin{tabular}{lcc}
\hline Descritores & CP1 & CP2 \\
\hline Nuesca & $-0,514216$ & $-0,677850$ \\
\hline Comraq & $-0,626116$ & $-0,019548$ \\
\hline Distan & 0,031917 & 0,904945 \\
\hline Pesfru & $-0,903428$ & 0,207938 \\
\hline Compca & 0,214498 & 0,081416 \\
\hline Espcas & $-0,901844$ & 0,108875 \\
\hline Facilde & 0,910060 & 0,157317 \\
\hline Pescas & $-0,896763$ & $-0,106279$ \\
\hline Texpol & $-0,248301$ & 0,402057 \\
\hline Sabfru & 0,823996 & $-0,132232$ \\
\hline Espend & $-0,905200$ & 0,219532 \\
\hline Peseme & $-0,346412$ & 0,735141 \\
\hline Água & $-0,272733$ & 0,348962 \\
\hline Óleo & $-0,414689$ & $-0,539043$ \\
\hline Fibras & $-0,295544$ & $-0,589420$ \\
\hline Variância $(\%)$ & $39,9 \%$ & $19,4 \%$ \\
\hline
\end{tabular}

As correlações entre os descritores, número de espigas por cacho (Nuesca), comprimento da ráquis (Comraq), peso do fruto (Pesfru), espessura das cascas (Espcas), peso das cascas (Pescas) e espessura da polpa (Espend) e o primeiro componente são negativas, indicando que as populações localizadas à esquerda do eixo x possuem cachos com maior número de espigas, cachos com maior comprimento de ráquis, bem como frutos mais pesados, cascas mais grossas e mais pesadas e maiores espessuras de polpa quando comparados com frutos das populações localizadas à direita do eixo x (Figura 2).

As correlações entre os descritores, facilidade para descascar os frutos (Facilde) e sabor do fruto são positivas, indicando que as populações à direita do eixo $\mathrm{x}$ possuem frutos fáceis de serem descascados (frutos pequenos) e mais saborosos do que aqueles das popula- 
ções localizadas à esquerda do eixo x. No segundo componente principal, destacam-se: distância morfológica do fruto (Distan) e peso da semente (Peseme) com correlações positivas e número de espigas por cacho (Nuesca) com correlação negativa, indicando que populações localizadas mais acima possuem frutos mais alongados com sementes mais pesadas e menor número de espigas por cacho do que aqueles frutos das populações localizadas mais abaixo.

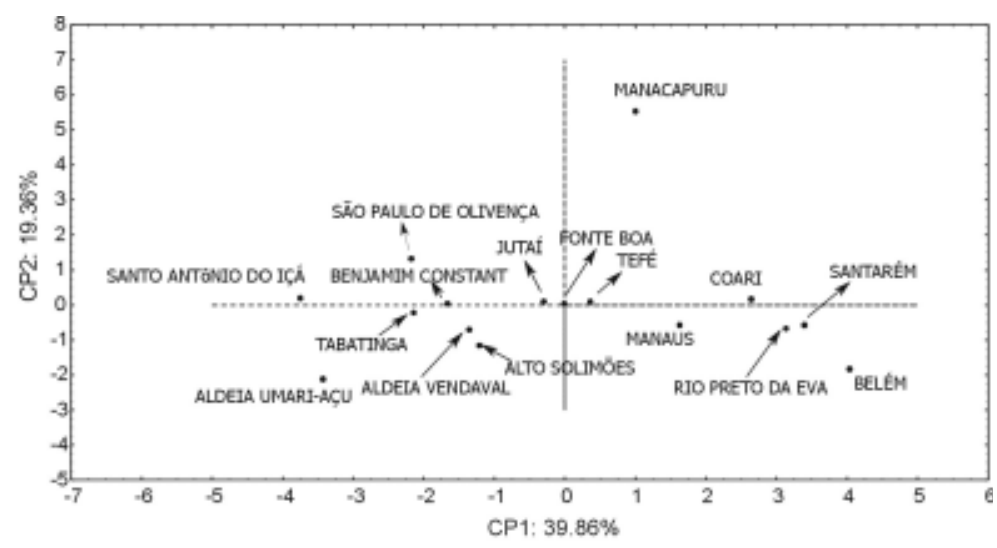

FIGURA 2 - Dispersão bidimensional segundo os componentes principais das 16 populações de pupunha das raças Pará, Putumayo e Solimões.

Nota-se, na Figura 2, que as populações localizadas à direita do eixo x: Belém, Santarém, Rio Preto da Eva, Manaus e Coari compõem exatamente o grupo 1, formado pela análise de agrupamento, tendo frutos com maior facilidade de serem descascados, frutos mais saborosos, frutos com menor peso, cascas mais finas, cascas mais leves, com menor espessura de polpa, menor número de espigas por cacho e menor comprimento de ráquis. Ainda na Figura 2, à esquerda do eixo x, estão localizadas as populações: Vendaval, Alto Solimões, Tabatinga, São Paulo de Olivença, Benjamim Constant e Umari-açu que compõem exatamente o grupo $2 \mathrm{da}$ análise de agrupamento. Por se localizarem à esquerda do eixo x, esse grupo possui frutos mais pesados, cascas mais grossas e mais pesadas, polpa mais grossa, maior dificuldade para serem descascados, frutos com menor sabor, frutos com maior número de espigas por cacho e maior comprimento de ráquis. $\mathrm{O}$ grupo 2 é um grupo intermediário entre o grupo $1 \mathrm{e}$ o grupo 3, concordando com Mora Urpi \& Clement (1988).

Em relação ao eixo y, nota-se, na Figura 2, destaque apenas para a população de Manacapuru por localizar-se bem acima das demais. Essa população possui frutos mais alongados e com maior peso em suas sementes, quando comparados às demais populações.

As populações de Manacapuru e Santo Antônio do Içá são populações atípicas nestas análises. $\mathrm{Na}$ análise de agrupamento, elas não pertenceram a nenhum grupo e, na análise de componentes principais, Manacapuru localiza-se no ponto mais alto do gráfico, enquanto Santo Antônio do Içá, no ponto mais extremo à esquerda.

A análise discriminante foi realizada com dez descritores: número de espigas por cacho (Nuesca), comprimento da ráquis (Comraq), peso do fruto (Pesfru), espessura das cascas (Espcas), peso das cascas (Pescas), espessura da polpa (Espend), facilidade para descascar os frutos (Facilde), sabor do fruto (Sabfru), distância morfológica do fruto (Distan) e peso da semente (Peseme) por apresentarem maior poder discriminante. As duas funções discriminantes (raiz 1 e raiz 2), mostrada na (Figura 3), expressaram $100 \%$ da variância, estando a contribuição dos descritores relacionada ao tamanho, qualidade e forma dos frutos. Estes resultados permitiram classificar as raças enquanto Mora-Urpi \& Clement (1985; 1988) e Mora-Urpi et al. (1997) utilizaram apenas o tamanho de frutos para discriminar a existência da raça Solimões. Entretanto, Clement (1986) utilizou descritores morfológicos das plantas e dos frutos, não conseguindo discriminar a raça Solimões das demais. A população de Fonte Boa, segundo o autor, seria muito similar à Putumayo, podendo ser uma transição entre esta e Solimões, e que, neste estudo, esta população ficou caracterizada como pertencente à raça Solimões.

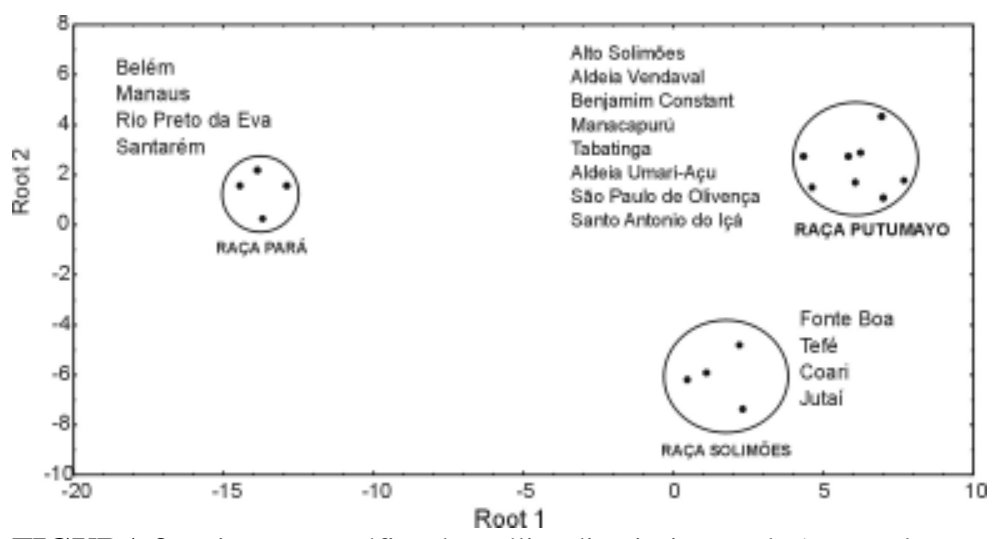

FIGURA 3 - Dispersão gráfica da análise discriminante de 16 populações de pupunha das raças Pará, Putumayo e Solimões

Também estes dados discordam de resultados obtidos com técnicas moleculares e isoenzimas (Picanço et al., 1999; Gallego Rodrigues et al.,1999; Rodrigues, 2001; Clement et al., 2002; Sousa et al., 2002), onde concluíram que a raça Solimões não existe. As três técnicas estatísticas multivariadas mostraram, em conjunto, ser um método eficiente de discriminação das raças Pará, Putumayo e Solimões.

\section{AGRADECIMENTOS}

Aos funcionários e técnicos do INPA, que nos auxiliaram nas caracterizações, e ao Dr. Charles Clement, pelo auxílio na metodologia.

\section{REFERÊNCIAS BIBLIOGRÁFICAS}

CLEMENT, C. R. Descriptores minimos para el pejibaye (Bactris gasipaes H.B.K. y sus implicaciones filogenéticas. 128p. Dissertação (Mestrado em Biologia),Universidade de Costa Rica, San José, 1986.

Clement, C. R. Pupunha (Bactris gasipaes Kunth, Palmae). Jaboticabal: FUNEP, 2000. 48p. (Série Frutas Nativas).

CLEMENT, C. R.; SOUZA, N. R.; RODRIGUES, D. P.; ASTOLFI-FILHO, S.; NÚÑES MORENO, Y.; TORRES PASQUAL, V.; GALLEGO RODRIGUES, F. J. 2002 Use of AFLPs to distinguish landraces of pejibaye (Bactris gasipaes) in Brazilian Amazônia. Scientia Agrícola, Piracicaba, v. 59, n. 4, p. 749-753, 2002.

CRUZ, C. D.; REGAZZI, A. J. Modelos biométricos aplicados ao melhoramento genético. Viçosa: Universidade Federal de Viçosa, 1994. 390p.

ENGELMAN, L. Discriminant Analysis. In: WILKINSON, L. (Ed.) SYSTAT 7.0 Statistics. Chicago, 1997. 751p.

GALLEGO RODRIGUEZ, F. J.; NÚÑES MORENO, Y; PICANÇO, D. B.; SOUZA, N. R.; CLEMENT, C. R.; ASTOLFI FILHO, S. Utilización de AFLPs para estudiar razas primitivas de la palmera melocotón o pejibaye (Bactris gasipaes Kunth) en la Amazonía brasileña. In: I CONGRESO DE LA SOCIEDAD ESPAÑOLADE GENÉTICA, La Coruña, Espanha, 1999, Resumen... p.361.

IRIARTE MARTEL, J. H. Caracterização de germoplasma de pupunha (Bactris gasipaes Kunth) por descritores morfológicos, 90f. Tese (Doutorado em Genética e Melhoramento de Plantas) Faculdade de Ciências Agrárias e Veterinárias, Universidade Estadual Paulista, Jaboticabal, 2002.

KLEKA, W. R. Discriminant analysis: quantitative application in the social science. California: University of California, 1975, p.19-20.

MORA URPI, J. Polinización en Bactris gasipaes H.B.K. (Palmae): nota adicional. Revista de BiologiaTropical, San José, v.30, p.174-176, 1982.

MORA URPI, J. El pejibaye (Bactris gasipaes H.B.K.): origen, biologia floral y manejo agronómico. In: PALMERAS poco utilizadas de América Tropical. Turrialba: FAO/CATIE, 1984. p.118-160.

MORA URPI, J.; CLEMENT, C. R. Races and population of peach palm found in the Amazon basin. In: FINAL report: peach palm Germplasm 
Bank. INPA, Manaus.1985. p.107-141.

MORA URPI, J.; CLEMENT, C. R. Races and population of peach palm found in the Amazon basin. FINAL report: Peah Palm Germplasm Bank. INPA, Manaus, 1988. p.78-94.

MORA URPI, J.; WEBER, J. C.; CLEMENT, C. R. Peach palm (Bactris gasipaes Kunth). In: INTERNATIONAL Plant Genetic Resources Intitute: Roma, 1997. 83p.

MOURÃO, K. S. M.; BELTRATTI, C. M. Morphology and anatomy of developing fruits and seeds of (Mammea americana L Crusiaceae). Revista Brasileira de Biologia, São Carlos, v.60, n.4, p.15-25, 2000.

PICANÇO, D. B.; SOUZA, N. R.; CLEMENT, C. R.; NAGÃO, E. O; ASTOLFI FILHO, S. Discriminação de raças primitivas de pupunha (Bactris gasipaes) na amazônia brasileira com marcadores moleculares (RAPDs).In:CONGRESSO BRASILEIRODE GENÉTICA,45, Gramados-RS. 1999, Resumos...p.293.

RANZANI, G. Identificação e caracterização de alguns solos da Estação Experimental de Silvicultura Tropical do INPA. Acta Amazônica, Manaus, v.10, n.1, p.7-41, 1980.
RIBEIRO, M. N. G. Aspectos climatológicos de Manaus. Acta Amazônica, Manaus, v.6, n.2, p. 229-232, 1976.

RODRIGUES, D. P. Análise das morfo-raças primitivas de pupunha (Bactris gasipaes Kunth), mantidas no banco Ativo de Germoplasma, com marcadores moleculares RAPDS. 71f. Dissertação. (Mestrado em Biologia). Faculdade de Biologia, Universidade de Brasilia, 2001.

SOKAL, R. R. Statistical methods in systematics. Journal of Biology, San Francisco, n.40, p. 337-391, 1965.

SNEATH, P. H.; SOKAL, R. R. Numerical taxonomy: The principles and practice of numerical classification. San Francisco: W.H. Freeman, 1973.573p.

SOUZA, N. R.; RODRIGUES, D. P.; CLEMENT, C. R.; NAGÃO, E. O.; ASTOLFI FILHO, S. Discriminação de raças primitivas de pupunha (Bactris gasipaes) na amazônia brasileira por meio de marcadores moleculares (RAPDs). Acta Amazônica, Manaus, v.31, n.4, p.539$545,2002$.

TABACHNICK, B. G.; FIDELL, L. S. Using Multivariate Statistics. California: Harper \& Row, 1983. 445p. 